ed signal transduction cascades such as those coordinated by the ErbB class of receptor tyrosine kinases $(21,22)$. Yang et al.'s study (8) shows that variola and related viruses are dependent upon some of the same pathways that the host cell uses for growth and development. Inhibitors of the ErbB-1 pathway as well as other cell-signal transduction pathways required for viral replication represent a largely untapped source of potential antiviral drugs and merit further exploration.

\section{Acknowledgments}

The authors wish to thank Nancy Touchette, Gregory Folkers, and Bernard Moss for helpful discussions.

Address correspondence to: Anthony S. Fauci, National Institute of Allergy and Infectious Diseases, NIH, Building 31, Room 7A03, 9000 Rockville Pike, Bethesda, Maryland 20892-2520, USA. Phone: (301) 496-2263; Fax: (301) 496-4409; E-mail: afauci@niaid.nih.gov.

\footnotetext{
1. Lane, H.C., La Montagne, J., and Fauci, A.S. 2001 Bioterrorism: a clear and present danger. Nat. Med. 7:1271-1273.
}

2. [Anonymous]. 2002. NIAID biodefense research agenda for CDC category A agents. NIH Publication No. 03-5308. www.niaid.nih.gov/biodefense/ research/biotresearchagenda.pdf.

3. Mahy, B.W.J. 2003. An overview on the use of a viral pathogen as a bioterrorism agent: why smallpox? Antiviral Res. 57:1-5.

4. Smith, G.L., and McFadden, G. 2002. Smallpox: anything to declare? Nat. Rev. Immunol. 2:521-527.

5. Harrison, S.C., et al. 2004. Discovery of antivirals against smallpox. Proc. Natl. Acad. Sci. U. S. A. 101:11178-11192.

6. Ibarra, V., Blanco, J.R., Oteo, J.A., and Rosel, L. 2000. Efficacy of cidofovir in the treatment of recalcitrant molluscum contagiosum in an AIDS patient. Acta Derm. Venereol. 80:315-316.

7. Greene, W.C. 2004. The brightening future of HIV therapeutics. Nat. Immunol. 5:867-871.

8. Yang, H., et al. 2005. Antiviral chemotherapy facilitates control of poxvirus infections through inhibition of cellular signal transduction. J. Clin. Invest. 115:379-387. doi:10.1172/JCI200523220.

9. Kim, M., et al. 2004. Biochemical and functional analysis of smallpox growth factor (SPGF) and anti-SPGF monoclonal antibodies. J. Biol. Chem. 279:25838-25848.

10. Tzahar, E., et al. 1998. Pathogenic poxviruses reveal viral strategies to exploit the ErbB signaling network. EMBO J. 17:5948-5963.

11. Buller, R.M.L, Chakrabarti, S., Cooper, J.A., Twardzik, D.R., and Moss, B. 1988. Deletion of the vaccinia virus growth factor gene reduces virus virulence. J. Virol. 62:866-874.

12. Opgenorth, A., Nation, N., Graham, K., and McFadden, G. 1993. Transforming growth factor alpha,
Shope fibroma growth factor, and vaccinia growth factor can replace myxoma growth factor in the induction of myxomatosis in rabbits. Virology. 192:701-709.

13. Smith, G.L., and Law, M. 2004. The exit of Vaccinia virus from infected cells. Virus Res. 106:189-197.

14. Newsome, T.P., Scaplehorn, N., and Way, M. 2004. Src mediates a switch from microtubule- to actin-based motility of Vaccinia virus. Science. 306:124-129.

15. Hall, A. 2004. Src launches vaccinia. Science. 306:65-67.

16. Yarden, Y., and Sliwkowski, M.X. 2001. Untangling the ErbB signalling network. Nat. Rev. Mol. Cell. Biol. 2:127-137.

17. Hooper,J.W., Custer, D.M., and Thompson, E. 2003. Four-gene-combination DNA vaccine protects mice against a lethal vaccinia virus challenge and elicits appropriate antibody responses in nonhuman primates. Virology. 306:181-195.

18. Fogg, C., et al. 2004. Protective immunity to vaccinia virus induced by vaccination with multiple recombinant outer membrane proteins of intracellular and extracellular virions. J. Virol. 78:10230-10237.

19. Rizzardi, C.P., et al. 2002. Treatment of primary HIV-1 infection with cyclosporin A coupled with highly active antiretroviral therapy. J. Clin. Invest. 109:681-688. doi:10.1172/JCI200214522.

20. Chapuis, A.G., et al. 2000. Effects of mycophenolic acid on human immunodeficiency virus infection in vitro and in vivo. Nat. Med. 6:762-768.

21. Cockerill, G.S., and Lackey, K.E. 2002. Small molecule inhibitors of the class I receptor tyrosine kinase family. Curr. Top. Med. Chem. 2:1001-1010.

22. Wakeling, A.E. 2002. Epidermal growth factor receptor tyrosine kinase inhibitors. Curr. Opin. Pharmacol. 2:382-387.

\title{
Knock your SOCS off!
}

\section{Derek LeRoith ${ }^{1}$ and Peter Nissley}

1Diabetes Branch, National Institute of Diabetes and Digestive and Kidney Diseases (NIDDK) and

2Metabolism Branch, National Cancer Institute, NIH, Bethesda, Maryland, USA.

The growth hormone/IGF-1-signaling (GH/IGF-1-signaling) system is involved in numerous physiological processes during normal growth and development and also in the aging process. Understanding the regulation of this system is therefore of importance to the biologist. Studies conducted over the past decade have shown that the JAK/STAT pathways are involved in GH signaling to the nucleus. More recently, evidence has been presented that a member of the SOCS family, SOCS2, is a negative regulator of GH signaling. This story began several years ago with the dramatic demonstration of gigantism in the SOCS2-knockout mouse. A more specific definition of the role of SOCS2 in GH signaling is provided in this issue of the JCI (see the related article beginning on page 397) by the demonstration that the overgrowth phenotype of the SOCS2 ${ }^{-/-}$mouse is dependent upon the presence of endogenous GH and that administration of GH to mice lacking both endogenous GH and SOCS2 produced excessive growth.

Nonstandard abbreviations used: $\mathrm{GH}$, growth hormone; GHR, GH receptor; IGF-1R, IGF-1 receptor; PIAS, protein inhibitor of activated STAT; SHP1, Src homology 1-containing tyrosine phosphatase.

Conflict of interest: The authors have declared that no conflict of interest exists.

Citation for this article: J. Clin. Invest. 115:233-236 (2005). doi:10.1172/JCI200524228.
Cytokines regulate an array of biological processes by activating cell surface receptor complexes, a process that initially involves oligomerization and activation of the JAK family of tyrosine kinases. In turn, JAKs phosphorylate the cell surface receptor, and signaling proteins such as STATs are recruited to these phosphotyrosine sites on the receptor; the proximity of the STATs allows them to be phosphorylated by the JAKs (Figure 1). Dimerization of the phosphorylated STATs leads to nuclear migration and regulation of gene expression (1). To control excessive cytokine effects, the cytokine signal is negatively regulated by a number of proteins, including protein tyrosine phosphatases such as Src homology 1-containing tyrosine phosphatase (SHP1), protein inhibitor of activated STAT (PIAS), and SOCS (2). The latter family is comprised of cytokine-inducible $\mathrm{SH} 2$-containing protein and SOCS1-SOCS7. SOCSs are furthermore induced by cytokine signaling and therefore form a closed-loop, negativefeedback control mechanism (Figure 1).

\section{SOCS2, a new player in growth} hormone receptor signaling

While cytokines and their receptors have traditionally been the domain of immunol- 


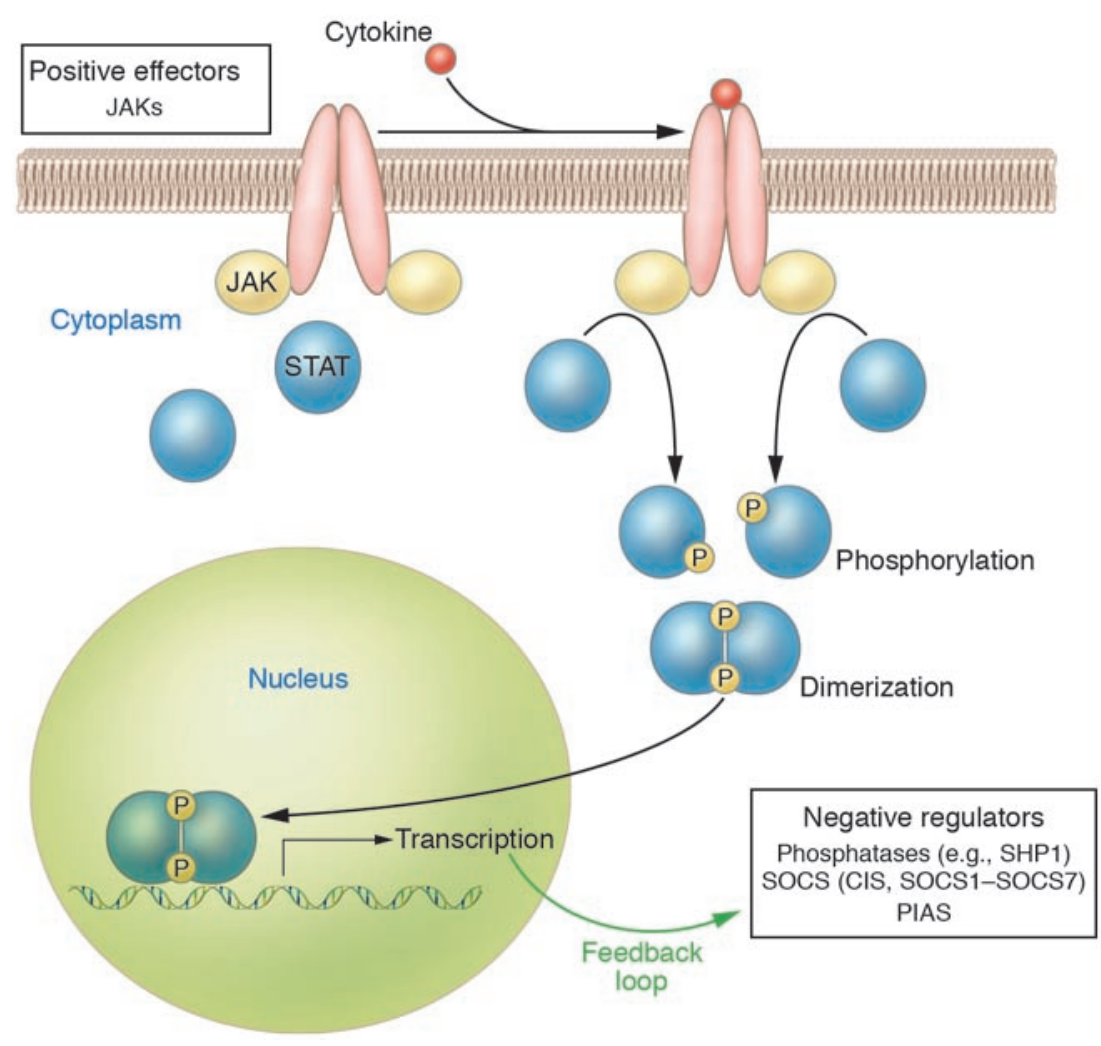

ogy, more recently their role in endocrinology has become apparent. The importance of the growth hormone/IGF-1 (GH/IGF-1) axis in clinical medicine is evidenced by GH/IGF-1 axis-related syndromes of excessive growth and short stature; the therapeutic efficacy of GH in treating $\mathrm{GH}$ deficiency and of IGF-1 in treating short stature secondary to mutations of the GH receptor (GHR) has been demonstrated. In a series of papers, including the report by Greenhalgh et al. in this issue of the JCI (3), investigators at the Walter and Eliza Hall Institute of Medical Research have defined a role for SOCS2 in regulating signaling by the GH/IGF-1 pathway. These investigators first reported that a SOCS2-knockout mouse exhibited gigantism (4). Since $\mathrm{SOCS}^{-/-}$mice resembled mice transgenic for GH (5) or for IGF-1 (6) and SOCS2 had been shown to be a negative regulator of GHR signaling in a cell culture model (7), the authors postulated that the $\mathrm{GH} /$ IGF-1 pathway was deregulated in the SOCS2 $2^{-/-}$mouse model. In SOCS2 $2^{-/-}$mice, IGF-1 mRNA levels are increased in the heart, lung, and spleen (4), perhaps concurrent with the relief of inhibition of GHR signaling. Surprisingly, Metcalf et al. (4) found that IGF-1 mRNA levels were not increased in the liver, the site of syn- thesis of circulating IGF-1 (8). Consistent with this observation is the finding that serum IGF-1 levels were not increased in SOCS2 $2^{--}$mice (4). However, serum IGF-1 levels have been reported to be increased in high-growth mice, in which a deletion breakpoint occurs within the SOCS2 gene (9). Since SOCS2 had been shown to bind to the IGF-1 receptor (IGF-1R) in yeast 2-hybrid experiments (10), Greenhalgh et al. speculated that relief of inhibition of IGF-1R signaling might also contribute to the overgrowth phenotype in the SOCS2 $2^{-/-}$ mouse (4). Efstratiadis and colleagues have provided genetic evidence that $\mathrm{GH}$ and IGF-1 promote postnatal growth by both independent and common functions, the common function reflecting the control of IGF-1 production by GH (11) (Figure 2).

The observations in the initial report on the SOCS2-knockout mouse were extended to show that primary cultures of hepatocytes from SOCS2-/- mice had moderately prolonged STAT5 signaling in response to GH (12). Furthermore, the increase in growth observed in $\mathrm{SOCS}^{-/-}$mice was attenuated when the SOCS2 gene was knocked out in mice also lacking STAT5b, consistent with the necessity of GHR activation of STAT5b for overgrowth in the SOCS2 mouse. In this issue of the JCI,

\section{Figure 1}

Model of JAK/STAT signaling and negative feedback by SOCS proteins. Cytokine signaling involves ligand binding and activation of the cell surface cytokine receptor. Recruitment and activation of JAK in turn facilitates phosphorylation of a STAT tyrosine residue, and subsequent STAT activation induces dimerization. This activation is tightly controlled by multiple negative regulators of phosphorylation such as phosphatases, SOCS, and PIAS. SOCS proteins are also induced by cytokine signaling and form a closed-loop, negative-feedback mechanism.

Greenhalgh et al. demonstrate conclusively that the SOCS2 $2^{-/-}$overgrowth phenotype is dependent on the overgrowth of endogenous GH (3). SOCS2 $2^{-/}$mice were crossed with lit/lit (little) mice that exhibit a point mutation in the GH-releasing hormone receptor and are GH deficient (13). The lit/lit mice demonstrated approximately $60 \%$ growth retardation as predicted, and the SOCS $^{-1-}$ mice showed postnatal enhanced growth compared to wild-type littermates. The SOCS2 ${ }^{-/-}$Ghrhr ${ }^{\text {lit } / l i t}$ mice demonstrated a growth phenotype indistinguishable from that of the Ghrbrit/lit mice. To test whether the SOCS2 phenotype is dependent on the GH-signaling pathway, GH was administered to the double mutants. This caused a distinct increase in growth (though not to normal levels) and duplicated the relative greater growth seen in SOCS2-/- mice compared with that in controls. While these observations appear clear-cut, paradoxically, marked overexpression of SOCS2 in a transgenic mouse also led to growth excess (14). Thus SOCS2 in fact acts as a dual effector molecule at varying concentrations as was also shown in in vitro studies (7). Theoretically this could be due to alterations in the expression of other SOCS molecules or related to the GHR-SOCS2 interaction. To investigate this, the authors 


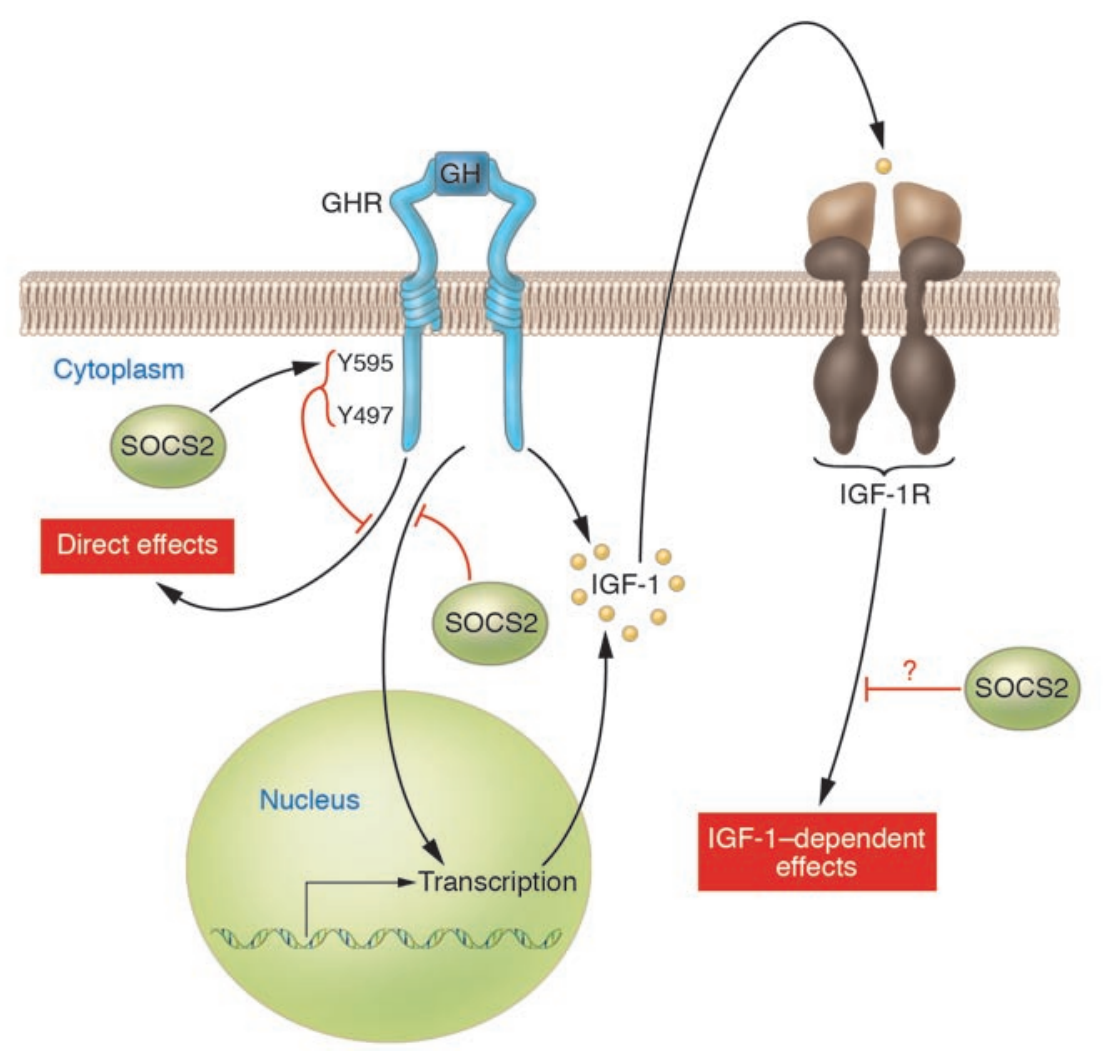

demonstrated by mutational analysis that Tyr595 and Tyr487 in the GHR are critical sites for SOCS2 interaction (3), sites previously shown to be important for binding of SHP2, a regulator of GH signaling $(15,16)$. Changing both tyrosines to phenylalanine resulted in a loss of SOCS2 inhibition at low concentrations of SOCS2 and loss of enhanced GHR signaling at high concentrations of SOCS2 whereas changing only Tyr487 appeared to primarily block the enhancement of GHR signaling seen at high SOCS concentrations.

\section{Unanswered questions remain}

While this paper sheds light on the role of SOCS2 protein in modulating GH signal transduction, a number of interesting questions remain to be answered.

Does SOCS2 negatively regulate IGF-1R function and thereby contribute to the SOCS2 $2^{-1-}$ overgrowth phenotype? Greenhalgh et al. previously reported (12) that IGF-1 did not induce SOCS2 expression in 3T3-F442A cells and that IGF-1R signaling was not altered in SOCS2 ${ }^{-/-}$primary embryo fibroblasts, which suggests that relief of inhibition of IGF-1R signaling does not contribute to the overgrowth phenotype of the SOCS2 $2^{-/-}$mouse. A limitation of this earlier experiment is that it was not dem- onstrated that wild-type primary embryo fibroblasts express SOCS2. More compelling evidence against regulation of IGF-1R signaling by SOCS 2 is perhaps provided by the growth patterns of SOCS2-/- mice (4). Since SOCS2 is expressed in mouse embryos (12) and IGF-1R has been shown to be important for fetal growth (17), it might be expected that $\mathrm{SOCS}^{-/-}$pups would be oversized. However, overgrowth in the SOCS2--mice was only seen in the current study after postnatal week 3 (3). Also, if relief of inhibition of IGF-1R by SOCS2 contributes in a major way to the SOCS2-/- overgrowth phenotype, then it would have been expected that the SOCS2 transgenic animal would have exhibited impaired growth rather than increased growth (14). On the other hand, overexpression of SOCS2 in MG-63 osteosarcoma cells elicited by a tetracycline-inducible system results in inhibition of IGF-1-stimulated MAPK activation (P. Nissley, unpublished observations). SOCS1, SOCS3, and SOCS6 have been shown to play a role in insulin resistance seen in states of cytokine overexpression by inhibiting the closely related insulin receptor tyrosine kinase and/or causing insulin receptor substrate-1 degradation $(18,19)$. Perhaps SOCS2 only negatively regulates IGF-1R signaling during states of cytokine

\section{Figure 2}

$\mathrm{GH}$ activates the GHR, which leads to multiple tissue effects, including IGF-1 gene expression. In turn, IGF-1, via IGF-1R, induces cellular effects. SOCS2 may negatively regulate these events at multiple levels. A series of papers from the Walter and Eliza Hall Institute of Medical Research, including the paper by Greenhalgh et al. in this issue of the $\mathrm{JCl}$ (3), provide convincing evidence that SOCS2 is a negative regulator of $\mathrm{GH}$ receptor signaling. Negative regulation of IGF-1R signaling by SOCS2 is more speculative. overexpression in which SOCS2 would be induced by cytokines.

What is the mechanism of SOCS2 inhibition (and enhancement) of GHR signaling? In this issue, Greenhalgh et al. (3) demonstrated that GHR Tyr595 and Tyr487 are important in SOCS2 binding to GHR. These tyrosines are also important for binding of SHP2. SHP2 has been shown to be a positive (15) or a negative regulator (16) of GHR signaling. Do SOCS2 and SHP2 compete for binding? It has been proposed that SOCS2 inhibits binding of STAT5 to GHR (20). Greenhalgh et al. show that the SOCSbox motif of SOCS2 is essential for negative regulation of GHR signaling (3). The SOCSbox motif has been shown to recruit SOCS proteins to an E3 ubiquitin ligase, which results in proteasomal degradation of SOCS-binding partners (19). Does SOCS2 binding to GHR result in receptor degradation? Finding an answer to this question will require further studies.

In summary, while the study by Greenhalgh et al. (3) supports the role of SOCS2 in GHR signaling, the mechanisms involved are yet to be fully determined. Nevertheless, understanding the regulation of GHR signaling and the production of IGF-1 will be critical in developing treatments for disorders of this system, including acromegaly (a 
result of excess $\mathrm{GH}$ ) and growth retardation, and perhaps even more importantly, treatment for the GH/IGF- 1 system that is active during acute illness and the aging process.

Address correspondence to: Derek LeRoith, Diabetes Branch, NIDDK, NIH, Room 8D12, Building 10, 9000 Rockville Pike, Bethesda, Maryland 20892-1758, USA. Phone: (301) 496-8090; Fax: (301) 4804386; E-mail: derek@helix.nih.gov.

1. Ihle, J.N. 1995. Cytokine receptor signalling. Nature. 377:591-594.

2. Fujimoto, M., and Naka, T. 2003. Regulation of cytokine signaling by SOCS family molecules. Trends Immunol. 24:659-666.

3. Greenhalgh, C.J., et al. 2005. Suppressor of cytokine signaling negatively regulates growth hormone action in vitro and in vivo. J. Clin. Invest. 115:397-407. doi:10.1172/JCI200522710.

4. Metcalf, D., et al. 2000. Gigantism in mice lacking suppressor of cytokine signalling-2. Nature. 405:1069-1073.

5. Palmiter, R.D., et al. 1982. Dramatic growth of mice that develop from eggs microinjected with metallothionein-growth hormone fusion genes.
Nature. 300:611-615.

6. Mathews, L.S., et al. 1988. Growth enhancement of transgenic mice expressing human insulin-like growth factor I. Endocrinology. 123:2827-2833.

7. Favre, H., Benhamou, A., Finidori, J., Kelly, P.A., and Edery, M. 1999. Dual effects of suppressor of cytokine signaling (SOCS-2) on growth hormone signal transduction. FEBS Lett. 453:63-66.

8. Yakar, S., et al. 1999. Normal growth and development in the absence of hepatic insulin-like growth factor I. Proc. Natl. Acad. Sci. U. S. A. 96:7324-7329.

9. Horvat, S., and Medrano, J.F. 2001. Lack of Socs2 expression causes the high-growth phenotype in mice. Genomics. 72:209-212.

10. Dey, B.R., Spence, S.L., Nissley, P., and Furlanetto, R.W. 1998. Interaction of human suppressor of cytokine signaling (SOCS)-2 with the insulin-like growth factor-I receptor. J. Biol. Chem. 273:24095-24101.

11. Lupu, F., Terwilliger, J.D., Lee, K., Segre, G.V., and Efstratiadis, A. 2001. Roles of growth hormone and insulin-like growth factor 1 in mouse postnatal growth. Dev. Biol. 229:141-162.

12. Greenhalgh, C.J., et al. 2002. Growth enhancement in suppressor of cytokine signaling 2 (SOCS-2)deficient mice is dependent on signal transducer and activator of transcription 5b (STAT5b). Mol. Endocrinol. 16:1394-1406.

13. Godfrey, P., et al. 1993. GHRH receptor of little mice contains a missense mutation in the extracellular domain that disrupts receptor function. Nat. Genet. 4:227-232.
14. Greenhalgh, C.J., et al. 2002. Biological evidence that SOCS-2 can act either as an enhancer or suppressor of growth hormone signaling. J. Biol. Chem. 277:40181-40184.

15. Kim, S.O., Jiang, J., Yi, W., Feng, G.S., and Frank, S.J. 1998. Involvement of the Src homology 2-containing tyrosine phosphatase SHP-2 in growth hormone signaling. J. Biol. Chem. 273:2344-2354.

16. Stofega, M.R., Herrington, J., Billestrup, N., and Carter-Su, C. 2000. Mutation of the SHP-2 binding site in growth hormone $(\mathrm{GH})$ receptor prolongs GH-promoted tyrosyl phosphorylation of GH receptor, JAK2, and STAT5B. Mol. Endocrinol. 14:1338-1350.

17. Liu, J.P., Baker, J., Perkins, A.S., Robertson, E.J., and Efstratiadis, A. 1993. Mice carrying null mutations of the genes encoding insulin-like growth factor I (Igf-1) and type 1 IGF receptor (Igf1r). Cell. 75:59-72.

18. Mooney, R.A., et al. 2001. Suppressors of cytokine signaling- 1 and -6 associate with and inhibit the insulin receptor. A potential mechanism for cytokine-mediated insulin resistance. J. Biol. Chem. 276:25889-25893.

19. Rui, L., Yuan, M., Frantz, D., Shoelson, S., and White, M.F. 2002. SOCS-1 and SOCS-3 block insulin signaling by ubiquitin-mediated degradation of IRS1 and IRS2. J. Biol. Chem. 277:42394-42398.

20. Ram, P.A., and Waxman, D.J.1999. SOCS/CIS protein inhibition of growth hormone-stimulated STAT5 signaling by multiple mechanisms. J. Biol. Chem. 274:35553-35561. 